\title{
DIAMETRIC THEOREMS IN SEQUENCE SPACES
}

\author{
R. AHLSWEDE, N. CAI and Z. ZHANG
}

Received January 4, 1989

\begin{abstract}
We determine in almost all Manhattan lattices configurations, which for specified diameter have maximal cardinality. Cases, in which those configurations are spheres, have been studied recently by Kleitman and Fellows.

For Hamming spaces we present a partial result supplementing a result of Frankl and Füredi and we formulate a general conjecture.
\end{abstract}

\section{Introduction}

In a finite metric space $(\Gamma, \mu)$ a (discrete) diametrical problem concerns a question like "How large can a subset $A$ of $\Gamma$ with $\mu\left(a, a^{\prime}\right) \leq \delta$ for all $a, a^{\prime} \in A$ be and what are the optimal configurations?"

For systems of subsets with the union function $d_{V}$ or binary sequences with the Hamming distance $d_{H}$ the diametrical problems have been solved by Katona [3] and Kleitman [4], respectively. Actually, it was observed in [1] that these two problems are equivalent. There are several notions of distances for general alphabets, which generalize the Hamming distance in the binary case. One of them is the Taxi metric (also called Manhattan metric or $L_{1}$-metric by some authors) $\mathcal{T}$, which for a sequence space $\mathscr{I}\left(\gamma^{n}\right)=\prod_{i=1}^{n} \mathscr{I}_{\gamma_{i}}$ with components $\mathscr{I}_{\gamma_{i}}=\left\{1,2, \ldots, \gamma_{i}\right\}$ assigns to two sequences $a^{n}=\left(a_{1}, \ldots, a_{n}\right)$ and $b^{n}=\left(b_{1}, \ldots, b_{n}\right)$ the distance

$$
\mathcal{G}\left(a^{n}, b^{n}\right)=\sum_{i=1}^{n}\left|a_{i}-b_{i}\right| .
$$

For any subset $A \subset \mathscr{Z}\left(\gamma^{n}\right)$ the diameter $D(A)$ is defined by

$$
D(A)=\max _{a^{n}, b^{n} \in A} \mathscr{T}\left(a^{n}, b^{n}\right)
$$

and the radius $R(A)$ is defined by

$$
R(A)=\min _{a^{n} \in A} \max _{b^{n} \in A} \mathscr{T}\left(a^{n}, b^{n}\right) .
$$

A great step towards the solution of the diameter problem for this metric was recently made by Kleitman and Fellows [6]: 
Theorem KF. In the case

$$
2 r+1 \leq \sum_{i=1}^{n}\left\lfloor\frac{\gamma_{i}}{2}\right\rfloor
$$

a maximal sized subset of $\mathscr{I}\left(\gamma^{n}\right)$ with radius $r$ has also the maximal possible size among subsets of $\mathscr{I}\left(\gamma^{n}\right)$ with diameter $2 r$.

Roughly speaking this result covers a quarter of the possibilities, because the cases where (1.4) is violated or the diameter is odd are not included.

With some new ideas we reduced the number of unsolved cases substantially. In particular in the important stationary case $\gamma_{1}=\gamma_{2}=\ldots=\gamma_{n}$ we have an essentially complete solution. The specific results are presented in Theorems $1, \ldots, 6$.

The following special spaces are important in our analysis. We write alphabets with an ODD number of elements in the form

$$
x_{\alpha}=\{-\alpha, \ldots,-1,0,1, \ldots, \alpha\}
$$

and for any sequence of positive integers $\alpha^{n}=\left(\alpha_{1}, \ldots, \alpha_{n}\right)$ we call

$$
x\left(\alpha^{n}\right)=x_{\alpha_{1}} \times \ldots \times x_{\alpha_{n}}
$$

the $\alpha^{n}$-space. Similarly, alphabets with an EvEN number of elements are written in the form

$$
y_{\beta}=\left\{-\left(\beta+\frac{1}{2}\right), \ldots,-\frac{1}{2}, \frac{1}{2}, \ldots,\left(\beta+\frac{1}{2}\right)\right\}
$$

and for any sequence of non-negative integers $\beta^{n}=\left(\beta_{1}, \ldots, \beta_{n}\right)$ we call

$$
y\left(\beta^{n}\right)=y_{\beta_{1}} \times \ldots \times y_{\beta_{n}}
$$

the $\beta^{n}$-space. Finally, we call the "mixed" case $\mathscr{X}\left(\alpha^{\ell}\right) \times y\left(\beta^{m}\right)$ the $\alpha^{\ell}-\beta^{m}$-space.

We are mainly interested in determining the quantity

$$
C\left(d, \alpha^{\ell}, \beta^{m}\right)=\max \left\{|A|: D(A) \leq d, A \subset \mathscr{X}\left(\alpha^{\ell}\right) \times \mathcal{Y}\left(\beta^{m}\right)\right\} .
$$

For $C\left(d, \alpha^{n}, \beta^{0}\right)$ and $C\left(d, \alpha^{0}, \beta^{n}\right)$ we use abbreviations $C\left(d, \alpha^{n}\right)$ and $C\left(d, \beta^{n}\right)$, resp. For $a^{n} \in \mathscr{X}\left(\alpha^{\ell}\right) \times \mathcal{Y}\left(\beta^{m}\right)$ we define the weight by the distance from the origin $O=(0, \ldots, 0)$, that is,

$$
\left\|a^{n}\right\|=\mathscr{T}\left(a^{n}, O\right)
$$

Notice that $O \in X\left(\alpha^{n}\right)$, but $O \notin Y\left(\beta^{n}\right)$.

At the end of the paper we present a result for Hamming metrics.

\section{2. $\alpha^{n}$-spaces}

We introduce the Taxi sphere around the origin

$$
S\left(r ; \alpha^{n}\right)=\left\{x^{n} \in \mathscr{X}\left(\alpha^{n}\right):\left\|x^{n}\right\| \leq r\right\}
$$

and denote its cardinality by $N\left(r ; \alpha^{n}\right)$. We begin with our simplest result. 


\section{Theorem 1.}

$$
C\left(2 r, \alpha^{n}\right)=N\left(r ; \alpha^{n}\right)
$$

Thus the sphere with center at the origin is an optimal configuration, if $d=2 r$ is even.

For the proof we need two kinds of auxiliary results.

\section{Numeric relations}

Let $M\left(k ; \alpha^{n}\right)$ be the number of integral solutions of the equation

$$
\sum_{i=1}^{n} z_{i}=k, 0 \leq z_{i} \leq \alpha_{i}, i=1,2, \ldots, n
$$

Obviously, for $\alpha=\sum_{i=1}^{n} \alpha_{i}$

$$
\sum_{k=0}^{\alpha} M\left(k ; \alpha^{n}\right) t^{k}=\prod_{i=1}^{n}\left(\sum_{j=0}^{\alpha_{i}} t^{j}\right)
$$

Lemma 1. For $\alpha^{n}=\alpha_{1} \alpha^{n-1}=\left(\alpha_{1}, \ldots, \alpha_{n}\right)$ we have

$$
\begin{aligned}
& N\left(r ; \alpha^{n}\right)=\sum_{i=-\alpha_{1}}^{\alpha_{1}} N\left(r-|i| ; \alpha^{n-1}\right) \\
& M\left(k ; \alpha^{n}\right)=\sum_{j=0}^{\alpha_{1}} M\left(k-j ; \alpha^{n-1}\right)
\end{aligned}
$$

and with the abbrevation $\alpha^{*}=\frac{1}{2} \sum_{i=1}^{n} \alpha_{i}$

$$
M\left(k ; \alpha^{n}\right) \leq M\left(k^{\prime} ; \alpha^{n}\right) \quad \text { iff } \quad\left|k-\alpha^{*}\right| \geq\left|k^{\prime}-\alpha^{*}\right| .
$$

In the stationary case $\alpha_{1}=\alpha_{2}=\ldots=\alpha_{n}$ ( $n \geq 2$ ) here strict inequality holds iff $\left|k-\alpha^{*}\right|>\left|k^{\prime}-\alpha^{*}\right|$.

Proof. (1) and (2) are obviously true. We prove (3) by induction. For $n=1(3)$ is true. In case $n=2$ we have

$$
M\left(k ; \alpha^{2}\right)= \begin{cases}k+1 & \text { for } 0 \leq k \leq \theta_{1} \\ \theta_{1}+1 & \text { for } \theta_{1} \leq k \leq \theta_{2} \\ \alpha_{1}+\alpha_{2}-k+1 & \text { for } \theta_{2} \leq k \leq \alpha_{1}+\alpha_{2}\end{cases}
$$

where $\theta_{1}=\min \left(\alpha_{1}, \alpha_{2}\right)$ and $\theta_{2}=\max \left(\alpha_{1}, \alpha_{2}\right)$.

Therefore all claims are justified for $n=2$.

Next notice that

$$
M\left(k ; \alpha^{n}\right)=M\left(k^{\prime} ; \alpha^{n}\right), \quad \text { if } \quad\left|k-\alpha^{*}\right|=\left|k^{\prime}-\alpha^{*}\right| .
$$


In case $k=k^{\prime}$ this is obvious and in case $k-\alpha^{*}=\alpha^{*}-k^{\prime}$ it follows from the fact that $\left(w_{1}, \ldots, w_{n}\right)$ is a solution of $\sum_{i=1}^{n} z_{i}=k$ exactly when $\left(\alpha_{1}-w_{1}, \ldots, \alpha_{n}-w_{n}\right)$ is a solution of $\sum_{i=1}^{n} z_{i}=k^{\prime}$. By (2.3) it suffices to show that

$$
M\left(k+1 ; \alpha^{n}\right) \leq M\left(k ; \alpha^{n}\right) \quad \text { for } \quad k \geq \alpha^{*}
$$

and that in the stationary case this inequality is strict. Now with (2) we obtain

$$
M\left(k+1 ; \alpha^{n}\right)-M\left(k ; \alpha^{n}\right)=M\left(k+1 ; \alpha^{n-1}\right)-M\left(k-\alpha_{1} ; \alpha^{n-1}\right)
$$

and the result follows inductively, because

$$
k+1-\frac{1}{2} \sum_{i=2}^{n} \alpha_{i}>k-\frac{1}{2} \sum_{i=2}^{n} \alpha_{i} \geq\left|k-\alpha_{1}-\frac{1}{2} \sum_{i=2}^{n} \alpha_{i}\right| .
$$

Here the last inequality holds, because $k \geq \frac{1}{2} \sum_{i=1}^{n} \alpha_{i}$ implies

$$
k-\frac{1}{2} \sum_{i=2}^{n} \alpha_{i} \geq-k+\alpha_{1}+\frac{1}{2} \sum_{i=2}^{n} \alpha_{i}
$$

\section{Pushing to the Center}

We define an order " $<_{c}$ " in $x_{\alpha}$ by

$$
x<_{c} y \text { iff either }|x|<|y| \text { or }|x|=|y| \text { and } x>0, y<0
$$

and an order " $\leq c$ " in $X\left(\alpha^{n}\right)$ by

$$
x^{n} \leq_{c} y^{n} \quad \text { iff } \quad x_{i} \leq_{c} y_{i} \text { for } i=1,2, \ldots, n .
$$

By means of this order we introduce the "pushing to the center operator" $P$ as follows:

For any set $A \subset X\left(\alpha^{n}\right)$ and any $x_{j}^{n}=\left(x_{1}, \ldots, x_{j-1}, x_{j+1}, \ldots, x_{n}\right) \in \prod_{1 \leq i \neq j \leq n} x_{\alpha_{i}}$ set

$$
A\left(x_{j}^{n}\right)=\left\{\left(z_{1}, \ldots, z_{n}\right) \in A: z_{i}=x_{i} \text { for } i \neq j\right\},
$$

let $P_{j} A\left(x_{j}^{n}\right)=\left\{\left(x_{1}, \ldots, x_{j-1}, x, x_{j+1}, \ldots, x_{n}\right): x\right.$ is one of the

$$
\left.\left|A\left(x_{j}^{n}\right)\right| \quad c \text {-smallest elements in } x_{\alpha_{j}}\right\}
$$

and finally let $P_{j} A=\bigcup_{x^{n}} P_{j} A\left(x_{j}^{n}\right)$.

If $P_{j} A=A$ for all $j$, then we say that $A$ is a $c$-down set. It is easy to verify the following important fact.

Lemma 2. Every $A \subset X\left(\alpha^{n}\right)$ can be pushed into a $c$-downset $A^{\prime}$ such that

$$
\begin{gathered}
|A|=\left|A^{\prime}\right| \\
D(A) \geq D\left(A^{\prime}\right) .
\end{gathered}
$$




\section{Proof of Theorem 1.}

The key idea in our proof is the use of the fact:

$$
\left\|x^{n}\right\|-\left\|y^{n}\right\| \equiv 0(\bmod 2) \text { implies } \mathcal{J}\left(x^{n}, y^{n}\right) \equiv 0(\bmod 2) .
$$

We proceed by induction on $n$. The case $n=1$ being trivial, let now $\alpha^{n}=\alpha_{1} \alpha^{n-1}$ and let $A \subset \mathscr{X}\left(\alpha^{n}\right)$ satisfy $D(A) \leq 2 r$. By Lemma 2 we can assume that $A$ is a $c$-downset. Therefore we have

$$
A_{u} \subset A_{v}, \quad \text { if } \quad u>_{c} v
$$

if $A_{u}=\left\{x^{n-1}: u x^{n-1} \in A\right\}$, and for every non-negative integer

$$
\theta \leq \alpha_{1}
$$

we have

$$
A_{-\theta} \subset A_{\theta}
$$

Consider now the sets

and define

$$
\begin{aligned}
& A_{\theta}^{0}=\left\{x^{n-1}:\left\|x^{n-1}\right\|\right. \text { is odd, } \\
& \left.A_{\theta}^{e}=\left\{x^{n-1}:\left\|x^{n-1}\right\| \text { is even, } x^{n-1} \in A_{\theta} \backslash A_{-\theta}\right\} \backslash A_{-\theta}\right\}
\end{aligned}
$$

We then have

$$
A_{-\theta}^{*}=A_{-\theta} \cup A_{\theta}^{0}, \quad A_{\theta}^{*}=A_{\theta} \backslash A_{\theta}^{0}=A_{-\theta} \cup A_{\theta}^{e}
$$

where here and elsewhere we define

$$
E(U, V)=\max _{u \in U, v \in V} \mathcal{J}(u, v)
$$

We shall show next that

$$
D\left(A_{-\theta}^{*}\right) \leq 2(r-\theta)
$$

For this notice that for

$$
a^{n-1}, b^{n-1} \in A_{-\theta} \subset A_{\theta} \text { and } x^{n-1}, y^{n-1} \in A_{\theta}^{0}
$$

the following sequences are in the set $A$ :

$$
(-\theta) a^{n-1},(-\theta) b^{n-1}, \theta a^{n-1}, \theta b^{n-1}, \theta x^{n-1}, \theta y^{n-1},(-\theta+1) x^{n-1} \text { and }(-\theta+1) y^{n-1} \text {. }
$$

From the fact $D(A) \leq 2 r$ we obtain therefore the inequalities

$$
\begin{aligned}
& \mathcal{J}\left(a^{n-1}, b^{n-1}\right), \mathcal{T}\left(a^{n-1}, x^{n-1}\right) \leq 2(r-\theta) \\
& \mathscr{T}\left(x^{n-1}, y^{n-1}\right) \leq 2(r-\theta)+1
\end{aligned}
$$

and

However, since $\left\|x^{n-1}\right\|$ and $\left\|y^{n-1}\right\|$ are odd, by (2.9) $\mathscr{T}\left(x^{n-1}, y^{n-1}\right)$ must be even. This shows that actually

$$
\mathcal{T}\left(x^{n-1}, y^{n-1}\right) \leq 2(r-\theta)
$$

This, (2.17) and (2.15) imply (2.16).

Similarly, we can prove that

$$
D\left(A_{\theta}^{*}\right) \leq 2(r-\theta)
$$

By the induction hypothesis and (1) in Lemma 1 we conclude our proof with 


$$
|A|=\sum_{u=-\alpha_{1}}^{\alpha_{1}}\left|A_{u}\right|=\sum_{u=-\alpha_{1}}^{\alpha_{1}}\left|A_{u}^{*}\right| \leq \sum_{u=-\alpha_{1}}^{\alpha_{1}} N\left(r-|u| ; \alpha^{n-1}\right)=N\left(r ; \alpha^{n}\right) .
$$

We address now the case of an ODD diameter. Again we present a complete solution for $\alpha^{n}$-spaces.

For this we introduce suitable "2-half-spheres".

For $d=2 r+1$ and $\alpha^{n}=\alpha^{n-1} \alpha_{n}$ with $\alpha_{1} \geq \alpha_{i}$ for $i=2, \ldots, n$ we set

$$
S^{*}\left(d / 2 ; \alpha^{n}\right)=\left\{x^{n}: x_{1} \leq 0 \text { and }\left\|x^{n}\right\| \leq \text { ror } x_{1}>0 \text { and }\left\|x^{n}\right\| \leq r+1\right\} .
$$

Clearly

$$
D\left(S^{*}\left(d / 2 ; \alpha^{n}\right)\right)=d .
$$

Theorem 2. If we assume w.l.o.g. $\alpha_{1} \geq \alpha_{i}$ for $i=2, \ldots, n$ then we have for $d=2 r+1$ $C\left(2 r+1, \alpha^{n}\right)=\left|S^{*}\left(d / 2 ; \alpha^{n}\right)\right|$.

The proof has now two more ingredients, namely, a metric $\mathscr{V}^{*}$ associated with $\mathscr{J}$ and a "pushing from left to plus" operator (and their interplay).

\section{The metric $\mathcal{T}^{*}$}

We introduce $\mathcal{G}^{*}: \mathscr{X}\left(\alpha^{n}\right) \times \mathscr{X}\left(\alpha^{n}\right) \rightarrow \mathbf{R}_{+}$by

$$
\mathscr{J}^{*}\left(a^{n}, b^{n}\right)= \begin{cases}\max \left\{\mathcal{G}\left(a^{\prime n}, b^{\prime n}\right): a^{\prime n} \leq_{c} a^{n}, b^{\prime n} \leq_{c} b^{n}\right\}, & \text { if } a^{n} \neq b^{n} \\ 0, & \text { if } a^{n}=b^{n}\end{cases}
$$

and the diameter

$$
D^{*}(A)=\max \left\{\mathscr{I}^{*}\left(a^{n}, b^{n}\right): a^{n}, b^{n} \in A\right\} .
$$

Three basic facts are readily verified.

\section{Lemma 3.}

(1) $\mathscr{I}^{*}\left(a^{n}, b^{n}\right)=\left\|a^{n}\right\|+\left\|b^{n}\right\|-\left|\left\{i: a_{i}>0, b_{i}>0\right\}\right|$

(2) $g^{*}$ is a metric.

(3) $D^{*}(\mu(A))=D(A)$ for a c-down set $A \subset \mathscr{X}\left(\alpha^{n}\right)$, where $\mu(A)$ is the set of $c$-maximal elements in $A$.

\section{Pushing Left to Plus}

We can assume that $\alpha_{1} \geq \alpha_{2} \geq \ldots \geq \alpha_{n}$. The operator below is based on the mapping $\varphi: x_{\alpha_{n-1}} \times x_{\alpha_{n}} \rightarrow x_{\alpha_{n-1}} \times x_{\alpha_{n}}$ defined by

$$
\varphi(x, y)= \begin{cases}(-x,-y) & \text { if } x<0, y>0 \\ (-x+1,-y) & \text { if } x>0, y>0 \\ (y, 0) & \text { if } x=0, y>0 \\ (x, y) & \text { otherwise. }\end{cases}
$$

It can be used to define for any $A \subset x\left(\alpha^{n}\right)$ a mapping $\phi: A \rightarrow \mathscr{X}\left(\alpha^{n}\right)$ by

$$
\phi\left(a^{n}\right)= \begin{cases}a^{n} & \text { if } a_{n}>0 \text { and } a^{n-2} \varphi\left(a_{n-1}, a_{n}\right) \in A \\ a^{n-2} \varphi\left(a_{n-1}, a_{n}\right) & \text { otherwise. }\end{cases}
$$

We also write $\phi(A)=\left\{\phi\left(a^{n}\right): a^{n} \in A\right\}$. 
For any set $B \subset \mathscr{X}\left(\alpha^{n}\right)$ we introduce the associated $c$-downset $\mathscr{D}(B)=\left\{x^{n}\right.$ : $\exists b^{n} \in B$ with $\left.x^{n} \leq_{c} b^{n}\right\}$.

Now we define the "pushing left to plus" operator $Q\left(=Q_{n-1, n}\right)$ by

$$
Q A=\mathscr{D}(\phi(A))
$$

Clearly,

$$
|Q A| \geq|\phi(A)|=|A| .
$$

We summarize further properties, which follow immediately from the definitions.

Lemma 4. For any set $A \subset X\left(\alpha^{n}\right)$

(1) $\mu(Q A)=\mu(\phi(A)) \subset \phi(A) \subset Q(A)$

(2) $a^{n-2} a_{n-1} a_{n} \in \phi(A)$ implies $a^{n-2} \varphi\left(a_{n-1}, a_{n}\right) \in \phi(A)$.

Lemma 5. For a $c$-downset $A D(Q A) \leq D(A)$.

Proof. By (3) in Lemma 3

$$
D(Q A)=D^{*}(\mathcal{M}(Q A))=D^{*}(\mathcal{M}(\phi(A))) \leq D^{*}(\phi(A))
$$

and since $A$ is a $c$-downset also

$$
D(A)=D^{*}(A) \text {. }
$$

It suffices therefore to show that $D^{*}(\phi(A)) \leq D^{*}(A)$ or that

$$
g^{*}\left(\phi\left(a^{n}\right), \phi\left(b^{n}\right)\right) \leq D^{*}(A) \text { for } a^{n}, b^{n} \in A .
$$

In the case that $\phi\left(a^{n}\right)=a^{n}, \phi\left(b^{n}\right)=b^{n}$, which includes the case $a_{n} \leq 0, b_{n} \leq 0$, this is of course true.

In the case $a_{n} \leq 0, b_{n}>0$ we notice that $\phi$ does not increase $\|\cdot\|$ and only in the case that $b_{n-1}>0 \phi$ may decrease $\left|\left\{i: a_{i}>0, b_{i}>0\right\}\right|$, but by at most 1 . Furthermore, in the case $b_{n-1}>0, b_{n}>0$ we have $\left\|\phi\left(b^{n}\right)\right\|=\left\|b^{n}\right\|-1$. Therefore by (1) in Lemma 3 we obtain

$$
\mathscr{I}^{*}\left(\phi\left(a^{n}\right), \phi\left(b^{n}\right)\right) \leq \mathscr{T}^{*}\left(a^{n}, b^{n}\right)
$$

and thus (2.27).

The case $a_{n}>0, b_{n} \leq 0$ being symmetrically the same, we are left with the case $a_{n}>0, b_{n}>0$ and (again by symmetry) $\phi\left(b^{n}\right) \neq b^{n}$. We devide this into two subcases.

Subcase $\phi\left(a^{n}\right) \neq a^{n}$ :

We establish (2.27) by verifying $\mathcal{I}^{*}\left(\phi\left(a^{n}\right), \phi\left(b^{n}\right)\right)=\mathcal{I}^{*}\left(a^{n}, b^{n}\right)$ with the following table for

$$
\mathscr{I}^{*}\left(a^{n}, b^{n}\right)-\mathcal{I}^{*}\left(a^{n-2}, b^{n-2}\right) \text { and } \mathcal{T}\left(\phi\left(a^{n}\right), \phi\left(b^{n}\right)\right)-\mathcal{I}^{*}\left(a^{n-2}, b^{n-2}\right):
$$

\begin{tabular}{|c|c|c|c|}
\hline$b_{n-1}$ & $<0$ & $=0$ & $>0$ \\
\hline$<0$ & $\Delta-1$ & $\Delta-1$ & $\Delta-1$ \\
$=0$ & $\Delta-1$ & $\Delta-1$ & $\Delta-1$ \\
$>0$ & $\Delta-1$ & $\Delta-1$ & $\Delta-2$ \\
\hline
\end{tabular}


Subcase $\phi\left(a^{n}\right)=a^{n}$ :

Here necessarily $\tilde{a}^{n}=a^{n-2} \varphi\left(a_{n-1}, a_{n}\right) \in A$.

We can easily verify $\mathcal{I}^{*}\left(\phi\left(a^{n}\right), \phi\left(b^{n}\right)\right)=\mathscr{I}^{*}\left(\tilde{a}^{n}, b^{n}\right)$ by the next table for

$$
\begin{aligned}
& \mathscr{T}^{*}\left(\phi\left(a^{n}\right), \phi\left(b^{n}\right)\right)-\mathscr{T}^{*}\left(a^{n-2}, b^{n-2}\right) \text { and } \mathscr{T}^{*}\left(\tilde{a}^{n}, b^{n}\right)-\mathscr{T}^{*}\left(a^{n-2}, b^{n-2}\right):
\end{aligned}
$$

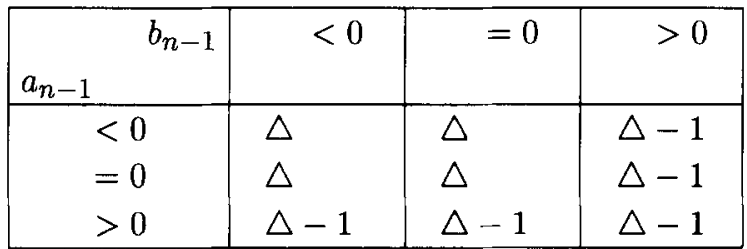

$$
\begin{aligned}
& \triangle=\left|a_{n-1}\right|+\left|a_{n}\right|+\left|b_{n-1}\right|+\left|b_{n}\right| \text {. }
\end{aligned}
$$

\section{Proof of Theorem 2}

We proceed by induction on $n$. The case $n=1$ is clear. By Lemmas 3, 4 and 5 we can assume that $A$ is a $c$-downset with the property

$$
a^{n}=a^{n-2} a_{n-1} a_{n} \in M(A) \text { implies } a^{n-2} \varphi\left(a_{n-1}, a_{n}\right) \in A .
$$

Let $A_{x}=\left\{x^{n-1}: x_{n-1} x \in A\right\}$ and consider for $\theta>0$ the sets

$$
\begin{aligned}
A_{\theta}^{+} & =\left\{x^{n-2} x_{n-1} \in A_{\theta} \backslash A_{-\theta}: x_{n-1}>0\right\} \\
A_{\theta}^{-} & =\left\{x^{n-2} x_{n-1} \in A_{\theta} \backslash A_{-\theta}: x_{n-1} \leq 0\right\} \\
A_{\theta}^{*} & =A_{\theta} \backslash A_{\theta}^{-}=A_{-\theta} \cup A_{\theta}^{+} \\
A_{-\theta}^{*} & =A_{-\theta} \cup A_{\theta}^{-}, A_{0}^{*}=A_{0} .
\end{aligned}
$$

Since $A$ is a $c$-downset, we have $A_{\theta} \supset A_{-\theta}$. Therefore for $a^{n-1}, b^{n-1} \in A_{-\theta} \subset A_{\theta}$ and $x^{n-1} \in A_{\theta}$ we have also $a^{n-1}(-\theta), b^{n-1} \theta, x^{n-1} \theta \in A$ and thus

$$
\mathscr{T}\left(a^{n-1}, b^{n-1}\right), \mathscr{J}\left(a^{n-1}, x^{n-1}\right) \leq d-2 \theta
$$

and

$$
D\left(A_{-\theta}\right), E\left(A_{-\theta}, A_{\theta}^{-}\right), E\left(A_{-\theta}, A_{-\theta}^{+}\right) \leq d-2 \theta .
$$

Now we are going to prove that also

$$
\begin{aligned}
& D\left(A_{\theta}^{-}\right)=D^{*}\left(\mu\left(A_{\theta}^{-}\right)\right) \leq d-2 \theta \\
& D\left(A_{\theta}^{+}\right)=D^{*}\left(\mu\left(A_{\theta}^{+}\right)\right) \leq d-2 \theta .
\end{aligned}
$$

Suppose (2.31) is not true. Then for some $a^{n-1}, b^{n-1} \in \mathcal{M}\left(A_{\theta}^{-}\right)$

$$
g^{*}\left(a^{n-1}, b^{n-1}\right)>d-2 \theta \text {. }
$$

Since $a^{n-1} \notin A_{-\theta}$ and $a^{n-1} \theta \in \mathcal{M}(A)$ we have $a^{n-2} \varphi\left(a_{n-1}, \theta\right) \in A$ by $(2.29)$.

Moreover, since $a_{n-1} \leq 0$ and $\theta>0$ by our definitions

$$
\varphi\left(a_{n-1}, \theta\right)= \begin{cases}\left(-a_{n-1},-\theta\right) & \text { if } a_{n-1}<0 \\ (\theta, 0) & \text { if } a_{n-1}=0\end{cases}
$$

Thus, noticing that $\theta>0$ and $b_{n-1} \leq 0$ we can conclude that 


$$
\begin{aligned}
d & \geq D(A) \geq \mathcal{I}^{*}\left(a^{n-2} \varphi\left(a_{n-1}, \theta\right), b^{n-1} \theta\right) \\
& =\mathscr{T}^{*}\left(a^{n-2}, b^{n-2}\right)+\left|a_{n-1}\right|+\left|b_{n-1}\right|+2 \theta \\
& =\mathscr{I}^{*}\left(a^{n-1}, b^{n-1}\right)+2 \theta>d .
\end{aligned}
$$

This contradiction proves (2.31).

Now suppose that $(2.32)$ is not true, that is, for some $a^{n-1}, b^{n-1} \in \mathcal{M}\left(A_{\theta}^{+}\right)$ (2.33) holds. By the reasoning given before $a^{n-2} \varphi\left(a_{n-1}, \theta\right) \in A$. Now $\varphi\left(a_{n-1}, \theta\right)=$ $\left(-\left(a_{n-1}-1\right),-\theta\right)$, because $a_{n-1}>0$ and $\theta>0$ in this case. Thus we arrive again at a contradiction:

$$
\begin{aligned}
d \geq D(A) & \geq \mathscr{I}^{*}\left(a^{n-2} \varphi\left(a_{n-1}, \theta\right), b^{n-1} \theta\right) \\
& =\mathscr{T}^{*}\left(a^{n-2}, b^{n-2}\right)+\mathcal{T}^{*}\left(\varphi\left(a_{n-1}, \theta\right), b_{n-1} \theta\right) \\
& =\mathcal{I}^{*}\left(a^{n-2}, b^{n-2}\right)+\left|a_{n-1}\right|+\left|b_{n-1}\right|+2 \theta-1 \\
& =\mathscr{T}^{*}\left(a^{n-1}, b^{n-1}\right)+2 \theta>d .
\end{aligned}
$$

So (2.32) holds. From (2.30), (2.31) and (2.32) we conclude that

$$
D\left(A_{\ell}^{*}\right) \leq d-2|\ell| \quad \text { for all } \ell
$$

and by the induction hypothesis

Therefore

$$
\left|A_{\ell}^{*}\right| \leq\left|S^{*}\left(\frac{d-2|\ell|}{2} ; \alpha^{n-1}\right)\right|
$$

$$
|A| \leq \sum_{\ell=-\alpha_{n}}^{\alpha_{n}}\left|S^{*}\left(\frac{d-2|\ell|}{2} ; \alpha^{n-1}\right)\right|=S^{*}\left(\frac{d}{2} ; \alpha^{n}\right)
$$

\section{3. $\alpha^{\ell}-\beta^{m}$-spaces}

Recall the definition of $X\left(\alpha^{\ell}\right) \times y\left(\beta^{m}\right)$ and of $C\left(d, \alpha^{\ell}, \beta^{m}\right)$ in the Introduction. We always assume that $n=\ell+m$.

The spheres introduced in (2.1) can be generalized to

$$
S\left(r ; \alpha^{\ell}, \beta^{m}\right)=\left\{x^{n} \in \mathcal{X}\left(\alpha^{\ell}\right) \times y\left(\beta^{m}\right):\left\|x^{n}\right\| \leq r\right\},
$$

where $r$ is a non-negative real number.

Theorem 3. In the case

$$
d+1 \geq \sum_{i=1}^{\ell}\left(\alpha_{i}+1\right)+\sum_{i=1}^{m}\left(\beta_{i}+1\right)
$$

we have

$$
C\left(d, \alpha^{\ell}, \beta^{m}\right) \leq \frac{1}{2}\left[\left|S\left(\underline{\delta} ; \alpha^{\ell}, \beta^{m}\right)\right|+\left|S\left(\bar{\delta} ; \alpha^{\ell}, \beta^{m}\right)\right|\right]
$$


where $\underline{\delta}=\left\lfloor\frac{d-m}{2}\right\rfloor+\frac{m}{2}$ and $\bar{\delta}=\left\lceil\frac{d-m}{2}\right\rceil+\frac{m}{2}$.

The bound is best possible, if $d-m$ is even.

Moreover, if $\alpha_{1}-1=\ldots=\alpha_{\ell}-1=\beta_{1}=\ldots=\beta_{m}, n \geq 2$, if strict inequality holds in (3.2) and if $d-m$ is even, then $S\left(\frac{d}{2} ; \alpha^{\ell}, \beta^{m}\right)$ is the unique optimal configuration.

Proof. Suppose that $A \subset \mathscr{X}\left(\alpha^{\ell}\right) \times \mathcal{Y}\left(\beta^{m}\right)$ and $D(A) \leq d$. For $J \subset\{1,2, \ldots, \ell\}$ let $\Omega(J)=J \cup\{\ell+1, \ldots, n\}$ and for $I \subset \Omega(J)$ define

$$
\begin{aligned}
& B_{J}(I)=\left\{x^{n} \in \mathscr{X}\left(\alpha^{\ell}\right) \times \mathcal{Y}\left(\beta^{m}\right): x_{i}>0, x_{j}<0 \text { and } x_{t}=0\right. \\
& \text { for } \left.i \in I, j \in I^{c}(J) \text { and } x_{t} \notin \Omega(J)\right\},
\end{aligned}
$$

where $I^{c}(J)=\Omega(J) \backslash I$.

Clearly, for $x^{n} \in B_{J}(I)$ and $y^{n} \in B_{J}\left(I^{c}(J)\right)$

$$
\mathcal{T}\left(x^{n}, y^{n}\right)=\left\|x^{n}\right\|+\left\|y^{n}\right\| \text {. }
$$

Define

$$
s=\max \left\{\left\|x^{n}\right\|: x^{n} \in B(I) \cap A\right\} .
$$

For any $J, I$ by (3.3) we also know that $\left\|y^{n}\right\| \leq d-s$ for all $y^{n} \in B\left(I^{c}(J)\right) \cap A$. We can therefore by (3.4) conclude that $\left|B_{J}(I) \cap A\right|$ cannot exceed the number of integral solutions of the inequalities

$$
\sum_{j \in J} z_{j}+\sum_{i=\ell+1}^{n} z_{i} \leq s-|J|-\frac{m}{2}
$$

$0 \leq z_{j} \leq \alpha_{j}-1$ for $j \in J$, and $0 \leq z_{\ell+i} \leq \beta_{i}$ for $i=1,2, \ldots, m$.

Similar relations hold for $\left|B_{J}\left(I^{c}(J)\right) \cap A\right|$ with $s$ replaced by $d-s$. Recalling the function $M$ in Section 2, we get therefore

$$
\left|B_{J}(I) \cap A\right|+\left|B_{J}\left(I^{c}(J)\right) \cap A\right| \leq \sum_{0 \leq k \leq p} M\left(k ; r^{|J|+m}\right)+\sum_{0 \leq k \leq q-p} M\left(k ; r^{|J|+m}\right),
$$

where $p=s-|J|-\frac{m}{2}$ must be integral, $q=d-2|J|-m$,

$$
r^{|J|+m}=\left(\alpha_{j_{1}}-1, \ldots, \alpha_{j_{|J|}}-1, \beta_{1}, \ldots, \beta_{m}\right), \quad \text { and } \quad\left\{j_{1}, \ldots, j_{|J|}\right\}=J .
$$

We denote the expression at the right side in (3.5) by $L(p)$ and prove next that for $p \geq \frac{q}{2}=\frac{d-m}{2}-|J|, L(p)$ is non-increasing and that in case

$$
\begin{gathered}
\alpha_{1}-1=\ldots=\alpha_{\ell}-1=\beta_{1}=\ldots=\beta_{m}, \\
d+1>\sum_{j=1}^{\ell}\left(\alpha_{j}+1\right)+\sum_{i=1}^{m}\left(\beta_{i}+1\right) \quad L(p) \text { is decreasing. }
\end{gathered}
$$

We check the difference between two neighbours:

$$
L(p+1)-L(p)=M\left(p+1 ; r^{|J|+m}\right)-M\left(q-p ; r^{|J|+m}\right) .
$$

For $r^{*}=\frac{1}{2}\left(\sum_{j \in J}\left(\alpha_{j}-1\right)+\sum_{i=1}^{m} \beta_{i}\right)$ we have, by (3.2) 


$$
\begin{gathered}
(p+1)+(q-p)=d+1-2|J|-m \geq \sum_{j=1}^{\ell}\left(\alpha_{j}+1\right)+\sum_{j=1}^{m}\left(\beta_{i}+1\right)-2|J|-m \geq \\
\sum_{j \in J}\left(\alpha_{j}+1\right)+\sum_{i=1}^{m}\left(\beta_{i}+1\right)-2|J|-m=2 r^{*} .
\end{gathered}
$$

By assumption $p \geq \frac{q}{2}$ we conclude that

$$
p \geq q-p \text { and } p+1>r^{*} .
$$

Now either

$$
\left|(q-p)-r^{*}\right|=r^{*}-(q-p) \leq p+1-r^{*}=\left|p+1-r^{*}\right|
$$

and the inequality is strict if it is strict in (3.2) or

$$
\left|(q-p)-r^{*}\right|=(q-p)-r^{*}<p+1-r^{*} \text {. }
$$

By (3) in Lemma 1 we obtain $L(p+1) \leq L(p)$ and the inequality is strict in the situation described. W.l.o.g. we can assume $p \geq q$, because otherwise we can exchange $I$ and $I^{c}(J)$ to get the same equation as (3.5) but with $p \geq \frac{q}{2}$. So we have thus shown

$$
\left|B_{J}(I) \cap A\right|+\mid B_{J}\left(I^{c}(J) \cap A \mid \leq L\left(\left[\frac{q}{2}\right\rceil\right)\right.
$$

and

$$
\begin{aligned}
& |A| \leq \frac{1}{2} \sum_{J \subset\{1,2, \ldots, \ell\}} \sum_{I \subset \Omega(J)}\left[\sum_{0 \leq k \leq\left\lfloor\frac{d-m}{2}\right\rfloor-|J|} M\left(k ; r^{|J|+m}\right)\right. \\
& =\frac{1}{2}\left[\sum_{J \subset\{1,2, \ldots, \ell\}} \sum_{I \subset \Omega(J)}\left[\sum_{0 \leq k \leq\left[\frac{d-m}{2}\right\rceil-|J|} M\left(k ; r^{|J|+m}\right)\right]\right.
\end{aligned}
$$

$$
\left.\left.+\sum_{0 \leq k \leq \bar{\delta}-|J|-\frac{m}{2}} M\left(k ; r^{|J|+m}\right)\right]\right]
$$

$=\frac{1}{2}\left[\left|S\left(\underline{\delta} ; \alpha^{\ell}, \beta^{m}\right)\right|+\mid S\left(\bar{\delta}, \alpha^{\ell}, \beta^{m} \mid\right]\right.$.

When $d-m$ is even, then $\underline{\delta}=\bar{\delta}=\frac{d}{2}$ and the expression to the right side in (3.2) equals $\left|S\left(\frac{d}{2} ; \alpha^{\ell}, \beta^{m}\right)\right|$.

Since $D\left(S\left(\frac{d}{2} ; \alpha^{\ell}, \beta^{m}\right)\right)=d$, the bound is best possible in this case.

The uniqueness part in the Theorem 3 follows from the fact that under the circumstances described any other configuration allows the choice of at least one $I \subset \Omega(J), J \subset\{1,2, \ldots, \ell\}$ with a strict inequality in (3.6) and therefore in (3.2). 


\section{Remarks}

1.) When the space is not of type $y\left(\beta^{n}\right)$ and $d-m$ is odd, then the bound (3.3) need not be best possible. In fact, Theorem 2 shows it.

2.) For the $\alpha^{\ell}-\beta^{m}$-space with $\ell, m \geq 1$ Kleitman and Fellows condition (1.4) can be written in the form

$$
d+1 \leq \sum_{i=1}^{\ell} \alpha_{i}+\sum_{i=1}^{m}\left(\beta_{i}+1\right)
$$

Comparing this with condition (3.1) we notice that Theorem KF together with Theorem 3 still leave out some values for $d$. The gap increases with $\ell$.

\section{4. $\beta^{n}$-spaces}

For $\beta^{n}$-spaces the previous general results can be refined.

Theorem 4. Suppose that

$$
d+1 \geq \sum_{i=1}^{n}\left(\beta_{i}+1\right),
$$

then

$$
C\left(d, \beta^{n}\right)=\frac{1}{2}\left[S\left(\underline{\delta} ; \beta^{n}\right)|+| S\left(\bar{\delta} ; \beta^{n}\right) \mid\right]
$$

where $\underline{\delta}=\left\lfloor\frac{d-n}{2}\right\rfloor+\frac{n}{2}$ and $\bar{\delta}=\left\lceil\frac{d-n}{2}\right\rceil+\frac{n}{2}$.

Moreover, if in the stationary case $\beta_{1}=\ldots=\beta_{n}(n \geq 2)$ the inequality in (4.1) is strict, then in case that $d-n$ is even $S\left(\frac{d}{2}, \beta^{n}\right)$ is the unique optimal configuration and in case that $d-n$ is odd all optimal configurations $A$ satisfy

$$
S\left(\underline{\delta} ; \beta^{n}\right) \subset A \subset S\left(\bar{\delta} ; \beta^{n}\right) .
$$

Proof. By Theorem 3 it suffices to present a configuration reaching the bound in (4.2) and to prove (4.3) in the case $d-n$ is odd. The latter can be done in the same way as in the proof of the uniqueness part in Theorem 3 . So we just have to find the optimal configuration in the case $d-n$ is odd. Actually in the proof of Theorem 3 we have also given a construction for a set $A$. It only remains to be seen that $D(A) \leq d$.

In the construction the weight of the elements is at most $\left[\frac{d-n}{2}\right]+\frac{n}{2} \stackrel{\text { def }}{=} r^{*}$ and if two elements $x^{n} \in B(I), y^{n} \in B\left(I^{\prime}\right)$ have both weight $r^{*}$, then $I \neq\{1,2, \ldots, n\}-I^{\prime}$. Therefore

$$
\mathcal{T}\left(x^{n}, y^{n}\right) \leq 2 r^{*}-1=d .
$$

We draw now attention to the fact that Kleitman and Fellows [6] condition reads for $\beta^{n}$-space

$$
d+1 \leq \sum_{i=1}^{n}\left(\beta_{i}+1\right)
$$


Thus, combining Theorem KF with our Theorem 4 we get a solution of the diametrical problem for $\beta^{n}$-space, where not covered is only the case

$$
d+1<\sum_{i=1}^{n}\left(\beta_{i}+1\right) \text { and } d \text { is odd. }
$$

Next we settle also this case except for a few "boundary points". A new sphere enters the scene.

Assuming w.l.o.g. that $\beta_{n}=\min _{1 \leq i \leq n} \beta_{i}$ we define the sphere

$$
S^{* *}\left(\frac{d}{2} ; \beta^{n}\right)=\left\{x^{n} \in \mathcal{Y}\left(\beta^{n}\right): \mathscr{J}\left(x^{n}, \varepsilon^{n}\right) \leq \frac{d}{2}\right\}
$$

with center $\varepsilon^{n}=\left(\frac{1}{2}, \frac{1}{2}, \ldots, \frac{1}{2}, 0\right)$.

Theorem 5. Suppose that $d$ is odd and

$$
d+1 \leq \sum_{i=1}^{n}\left(\beta_{i}+1\right)-\beta_{n}
$$

then

$$
C\left(d, \beta^{n}\right)=\left|S^{* *}\left(\frac{d}{2} ; \beta^{n}\right)\right| .
$$

Proof. We use Theorem KF. It says that for $r^{\prime}=r-\frac{1}{2}$

$$
S^{\prime}\left(r^{\prime}, \beta^{n-1}\right) \stackrel{\text { def }}{=}\left\{x^{n-1} \in \mathcal{Y}\left(\beta^{n-1}\right): \mathcal{T}\left(x^{n-1}, \eta^{n-1}\right) \leq r^{\prime}\right\}
$$

with $\eta^{n-1}=\left(\frac{1}{2}, \ldots, \frac{1}{2}\right)$ is an optimal configuration in $\mathcal{Y}\left(\beta^{n-1}\right)$, if for $d=2 r^{\prime}=d-1$ $d^{\prime}+1 \leq \sum_{i=1}^{n-1}\left(\beta_{i}+1\right)$.

We assume now that $A$ is a $c$-downset with $D(A) \leq d$. Let

$$
\begin{aligned}
A_{x} & =\left\{x^{n-1}: x^{n-1}\left(\frac{x}{|x|}\left(|x|-\frac{1}{2}\right)\right) \in A\right\}, \\
A_{\theta}^{0} & =\left\{x^{n-1}:\left\|x^{n-1}\right\| \text { is odd and } x^{n-1} \in A_{\theta} \backslash A_{-\theta}\right\}, \\
A_{\theta}^{e} & =\left\{x^{n-1}:\left\|x^{n-1}\right\| \quad \text { is even and } x^{n-1} \in A_{\theta} \backslash A_{-\theta}\right\}, \\
A_{-\theta}^{*} & =A_{-\theta} \cup A_{\theta}^{0} \text { and } A_{\theta}^{*}=A_{\theta} \backslash A_{\theta}^{0}=A_{-\theta} \cup A_{\theta}^{e}
\end{aligned}
$$

for $\theta>0$. In the same way as in the proof of Theorem 2 we can get

$$
D\left(A_{-\theta}\right), E\left(A_{-\theta}, A_{\theta}\right) \leq d-(2 \theta-1) \text {. }
$$

Furthermore, for $a^{n-1}, b^{n-1} \in A_{\theta}^{0}$ with $\theta>1$ we have $a^{n-1}\left(\theta-\frac{1}{2}\right), b^{n-1}\left[-\left(\theta-\frac{3}{2}\right)\right] \in A$.

Hence,

$$
\mathcal{T}\left(a^{n-1}, b^{n-1}\right) \leq d-2(\theta-1) \text { for all } a^{n-1}, b^{n-1} \in A_{\theta}^{0}
$$


and $\theta>0$.

However, since $\left\|a^{n-1}\right\|$ and $\left\|b^{n-1}\right\|$ are odd, $\mathscr{T}\left(a^{n-1}, b^{n-1}\right)$ must be even. Recalling that $d$ is odd we obtain $\mathcal{G}\left(a^{n-1}, b^{n-1}\right) \leq d-(2 \theta-1)$ and thus $D\left(A_{\theta}^{0}\right) \leq d-(2 \theta-1)$.

This and (4.9) imply

$$
D\left(A_{-\theta}^{*}\right) \leq d-(2 \theta-1)
$$

Similarly we can prove that

$$
D\left(A_{\theta}^{*}\right) \leq d-(2 \theta-1) .
$$

These two inequalities, Theorem KF and recurrence relations imply (4.8).

\section{More about uniqueness}

We are concerned here with the uniqueness parts in Theorem 4 and Theorem 3.

Let us look first at $\beta^{n}$-spaces. For even diameter we have a complete solution. We observe that the optimal configurations are different in the cases $d+1 \leq \sum_{i=1}^{n}\left(\beta_{i}+1\right)$ and $d+1 \geq \sum_{i=1}^{n}\left(\beta_{i}+1\right)$. In both cases they are spheres, but with different centers. In the first case the centers are lattice points and in the second case they are not.

However, if $d+1=\sum_{i=1}^{n}\left(\beta_{i}+1\right)$, then both, Theorem KF and Theorem 4, apply and there are optimal configurations of both kinds. Actually, in the example below they are not isomorphic. This demonstrates that our uniqueness result in Theorem 4 is in a sense best possible.

Example. $n=2, \beta_{1}=1, \beta_{2}=2, d=4, r=2$
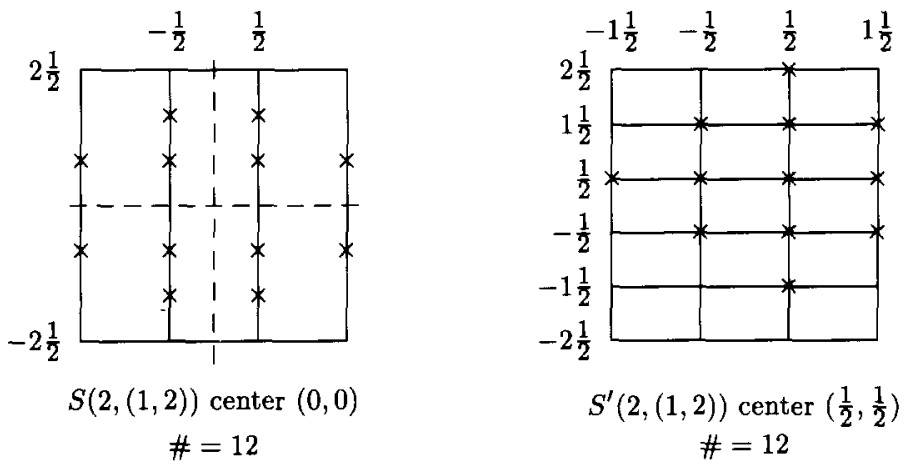

The following result show that the uniqueness result in Theorem 3 can be improved in so far as the lower bound condition on $d$ can be weakened. For this we consider the simplest $\alpha^{n}$-spaces, namely $x_{1}^{n}$.

Theorem 6. Suppose that for $d=2 r$

$$
3 d \geq 4 n
$$


then for $\alpha^{n}=(1,1, \ldots, 1) C\left(d, \alpha^{n}\right)$ is assumed only for $S\left(r, \alpha^{n}\right)$, that is, the sphere in $x_{1}^{n}$ of radius $r$ and center at the origin.

Proof. For all $I \subset\{1,2, \ldots, n\}$ define

$$
D(I)=\left\{x^{n}: x_{i} \in\{0,-1\} \text { for } i \in I \text { and } x_{i} \in\{0,1\} \text { for } i \in I\right\}
$$

and for an optimal $A L(I, k)=\left|\left\{x^{n}:\left\|x^{n}\right\|=k, x^{n} \in A \cap D(I)\right\}\right|$. Now we have for every $x^{n} \in X_{1}^{n}$ with $\left\|x^{n}\right\|=k$

$$
\left|\left\{I: x^{n} \in D(I)\right\}\right|=2^{n-k} .
$$

Therefore

$$
\begin{aligned}
|A| & =\sum_{I \subset\{1,2, \ldots, n\}} \sum_{k=0}^{n} L(I, k) 2^{-(n-k)} \\
& =\frac{1}{2} \sum_{I}\left[\sum_{k=0}^{n} L(I, k) 2^{-(n-k)}+\sum_{k=0}^{n} L\left(I^{c}, k\right) 2^{-(n-k)}\right] .
\end{aligned}
$$

For fixed $I$ define

$$
s=\max \left\{\left\|x^{n}\right\|: x^{n} \in A \cap D(I)\right\} \mid .
$$

For some $I$ we can assume that $s \geq \frac{d}{2}$.

Moreover, similar to the proof of Theorem 3 we can conclude that

$$
\left\|y^{n}\right\| \leq d-s \text { for all } y^{n} \in A \cap D\left(I^{c}\right)
$$

Thus

$$
\begin{aligned}
& \sum_{k=0}^{n} L(I, k) 2^{-(n-k)}+\sum_{k=0}^{n} L\left(I^{c}, k\right) 2^{-(n-k)} \\
& \leq 2^{-n}\left[\sum_{k=0}^{s}\left(\begin{array}{l}
n \\
k
\end{array}\right) 2^{k}+\sum_{k=0}^{d-s}\left(\begin{array}{l}
n \\
k
\end{array}\right) 2^{k}\right] \stackrel{\text { def }}{=} 2^{-n} F(s)
\end{aligned}
$$

By the definition of $F$ we obtain

$$
F(s+1)-F(s)=B(n, s+1)-B(n, d-s),
$$

where $B(n, k)=\left(\begin{array}{l}n \\ k\end{array}\right) 2^{k}$.

From (5.3) - (5.5) we know that the proof can be completed by showing that

$$
B(n, s+1)<B(n, d-s) \text { for } s \geq \frac{d}{2} .
$$

We prove it in two steps.

Step 1: We show that

$$
\frac{B(n, s+1)}{B(n, d-s-1)}<\frac{B(n, s+1)}{B(n, d-s)} \quad \text { for } \quad s \geq \frac{d}{2} \quad \text { and } \quad \frac{1}{2} d \leq n \leq \frac{3}{4} d \text {. }
$$


Since $(n+1)(d+1-n) \geq\left(\frac{3}{4} d+1\right)\left(\frac{1}{4} d+1\right)>\frac{3}{4}\left(\frac{d}{2}+1\right)^{2} \geq \frac{3}{4}(d-s)(s+2)$, i.e. $4(n-s-1)(n-d+s+1)<(s+2)(d-s)$. Thus

$$
\begin{aligned}
& \frac{B(n, s+2)}{B(n, d-s-1)}=\frac{\left(\begin{array}{c}
n \\
s+2
\end{array}\right) 2^{s+2}}{\left(\begin{array}{c}
n \\
d-s-1
\end{array}\right) 2^{d-s-1}}=4 \frac{\frac{n-s-1}{s+2}\left(\begin{array}{c}
n \\
s+1
\end{array}\right) 2^{s+1}}{\frac{d-s}{n-d+s+1}\left(\begin{array}{c}
n \\
d-s
\end{array}\right) 2^{d-s}} \\
& =\frac{4(n-s-1)(n-d+s+1)}{(s+2)(d-s)} \frac{B(n, s+1)}{B(n, d-s)}<\frac{B(n, s+1)}{B(n, d-s)} .
\end{aligned}
$$

Step 2: It remains to be seen that

$$
\begin{gathered}
\frac{B(n, s+1)}{B(n, d-s)}<1 \quad \text { when } \quad s=\frac{d}{2} \quad \text { and } 3 d \geq 4 n, \quad \text { i.e. } \\
\frac{B(n, r+1)}{B(n, r)}=\frac{\left(\begin{array}{c}
n \\
r+1
\end{array}\right) 2^{r+1}}{\left(\begin{array}{l}
n \\
r
\end{array}\right) 2^{d}}=\frac{2(n-r-1)}{r+1}<1,
\end{gathered}
$$

because $3 d \geq 4 n>4 n-6$.

Noticing that $d \leq 2 n$ we complete the proof.

\section{Remarks about the Hamming case}

The familiar Hamming metric over binary alphabet can also be generalized to general alphabets $\mathscr{I}_{\gamma}=\{1,2, \ldots, \gamma\}$. It is a map $d_{H}: \mathscr{I}_{\gamma}^{n} \times \mathscr{I}_{\gamma}^{n} \rightarrow\{0,1, \ldots, n\}$ defined by

$$
d_{H}\left(x^{n}, y^{n}\right)=\left|\left\{i \in\{1,2, \ldots, n\}: x_{i} \neq y_{i}\right\}\right| .
$$

What can be said about the diametrical problem for this metric, that is, about the function

$$
H_{\gamma}(n, d)=\max \left\{|A|: A \subset \mathscr{Q}_{\gamma}^{n}, d_{H}\left(a^{n}, b^{n}\right) \leq d \quad \text { for all } a^{n}, b^{n} \in A\right\} ?
$$

For "small values" of $n$ Frankl and Füredi [2] proved

Theorem FF. $H_{\gamma}(n, d)=\gamma^{d} \quad$ iff $\quad n \leq \gamma-1+d \quad$ or $\quad d=0,1$.

We announce here without proof a result for "big values" of $n$.

Theorem 7. For $n \geq(\gamma-1)^{d-1}+d$

$$
H_{\gamma}(n, d)= \begin{cases}\sum_{i=0}^{\underline{r}}\left(\begin{array}{c}
n \\
i
\end{array}\right)(\gamma-1)^{i} & \text { if } d \text { is even } \\
\sum_{i=0}^{\bar{r}}\left(\begin{array}{c}
n \\
i
\end{array}\right)(\gamma-1)^{i}+\left(\begin{array}{c}
n-1 \\
\underline{r}
\end{array}\right) & \text { if } d \text { is odd }\end{cases}
$$

where $\underline{r}=\left\lfloor\frac{d}{2}\right\rfloor$ and $\bar{r}=\left\lceil\frac{d}{2}\right\rceil$. 
For $\gamma=2$ this is a well-known result. As explained in [1] it is to be attributed to Kleitman [3] and to Katona [4]. Their methods can be generalized to yield our result. Whereas the optimal configuration in the case $n \leq \gamma-1+d$ is a cylinder it is a sphere for $n \geq(\alpha-1)^{d-1}+d$.

Examples show that for some middle $n$ 's both configurations are not optimal.

We suggest a mixture of cylinder and sphere as a candidate for an optimal configuration with diameter $d$ :

$$
C S(n, m, d)=\left\{a^{n}=a^{d-2 m} \cdot a^{n-d+2 m} \in \mathscr{I}_{\gamma}^{n}: d_{H}\left(a^{n-d+2 m}, O\right) \leq m\right\} .
$$

This becomes a cylinder when $m=0$ and a sphere when $d-2 m=0$. The cardinality of this configuration is

$$
\gamma^{d-2 m} \sum_{i=0}^{m}\left(\begin{array}{c}
n-d+2 m \\
i
\end{array}\right) \alpha^{i} .
$$

It can be maximized over $m \in\{0, \ldots, \underline{r}\}$.

\section{References}

[1] R. Ahlswede, and G. O. H. Katona: Contributions to the Geometry of Hamming Spaces, Disc. Math. 17 (1977), 1-22.

[2] P. FRANKL, and Z. FüredI: The Erdős-Ko-Rado Theorem for Integer Sequences, SIAM J. Alg. Disc. Meth. Vol 1, No. 4 (1980), 316-381.

[3] G. O. H. Katona: Intersection Theorems for Systems of Finite Sets, Acta Math. Acad. Sci. Hungar. 15 (1964), 329--337.

[4] D. J. Kleitman: On a Combinatorial Problem of Erdős, J. Comb. Theory 1 (1986), $209-214$.

[5] A. Moon: An Analogue of Erdös-Ko-Rado Theorem for the Hamming Schemes $H(n, q), J$. Comb. Theory A 32 (1982), 386-390.

[6] D. J. Kleitman, and M. Fellows: Radius and Diameter in Manhattan Lattices, Preprint 1986.

R. Ahlswede, N. Chai, Z. Zhang

Universität Bielefeld

Fakultät für Mathematik

Universitätsstraße 1

D-4800 Bielefeld

Germany. 